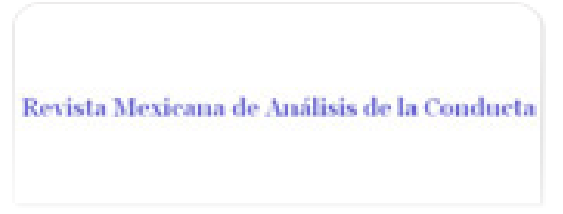

Revista Mexicana de Análisis de la Conducta ISSN: 0185-4534

editora@rmac-mx.org

Sociedad Mexicana de Análisis de la Conducta México

Mancilla Díaz, Juan Manuel; Escartín Pérez, Rodrigo Erick; López Alonso, Verónica Efectos de la 5-ht en ratas pretratadas con ketanserina sobre la estructura de la conducta alimentaria Revista Mexicana de Análisis de la Conducta, vol. 32, núm. 1, junio, 2006, pp. 55-71

Sociedad Mexicana de Análisis de la Conducta Guadalajara, México

Disponible en: http://www.redalyc.org/articulo.oa?id=59332105

- Cómo citar el artículo

- Número completo

- Más información del artículo

- Página de la revista en redalyc.org

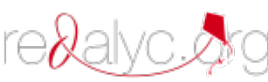

Sistema de Información Científica Red de Revistas Científicas de América Latina, el Caribe, España y Portugal Proyecto académico sin fines de lucro, desarrollado bajo la iniciativa de acceso abierto 


\title{
EFECTOS DE LA 5-HT EN RATAS PRETRATADAS CON KETANSERINA SOBRE LA ESTRUCTURA DE LA CONDUCTA ALIMENTARIA ${ }^{1}$
}

\author{
EFFECTS OF 5-HT IN KETANSERIN-PRETREATED RATS ON THE \\ STRUCTURE OF FEEDING BEHAVIOR \\ JUAN MANUEL MANCILLA DÍAZ² RODRIGO ERICK ESCARTÍN PÉREZ Y \\ VERÓNICA LÓPEZ ALONSO \\ LABORATORIO DE PSICOBIOLOGÍA DE LA ALIMENTACIÓN, \\ UNIVERSIDAD NACIONAL AUTÓNOMA DE MÉXICO, FES-IZTACALA.
}

\begin{abstract}
RESUMEN
Existe evidencia de que el control serotoninégico sobre la conducta alimentaria es mediado principalmente por receptores $5-\mathrm{HT}_{1 / 2}$. Sin embargo, no son suficientes los datos acerca de los mecanismos conductuales específicos involucrados. El propósito del presente estudio fue evaluar los efectos de la 5- $\mathrm{HT}$ en ratas pretratadas con ketanserina sobre la estructura de la conducta alimentaria con el fin de revelar la contribución de los receptores $5-\mathrm{HT}_{2 \mathrm{~A} / 2 \mathrm{C}}$ del núcleo paraventricular hipotalámico (NPH) y el mecanismo conductual específico involucrado. Cuarenta ratas fueron mantenidas bajo un paradigma de auto-selección dietaria con libre acceso a fuentes separadas de proteínas, carbohidratos, grasas y agua. La estructura de la conducta alimentaria fue registrada durante $20 \mathrm{~min}$. Las ratas se mantuvieron en una habitación a $21 \pm$ $1^{\circ} \mathrm{C}$, bajo un ciclo invertido de luz-oscuridad de $12 \mathrm{hrs}$. Los datos obtenidos indican que el pretratamiento con ketanserina atenuó el efecto supresor de la 5-HT sobre la ingesta de carbohidratos en el arranque del período natu-

1. Proyecto financiado por DGAPA, PAPIIT IN304300.

2. Correspondencia: Juan Manuel Mancilla-Díaz, Laboratorio de Psicobiología de la Alimentación, UNAM FES-Iztacala. Av de los Barrios \#1, Los Reyes Iztacala, Tlalnepantla Edo. de México. CP. 54090. Tel. + 52(55) 56231298, ext. 410, 409 y 457. Fax: +52(55) 5390 7604. E-mail: jmmd@servidor.unam.mx
\end{abstract}


ral de alimentación (oscuridad) e incrementó la duración de los periodos de ingestión de carbohidratos. El pretratamiento con ketanserina fue incapaz de prevenir el decremento sobre la tasa local de alimentación inducida por la 5-HT. Nuestros datos apoyan el argumento de que la estimulación de los receptores $5-\mathrm{HT}_{2 \mathrm{~A} / 2 \mathrm{C}}$ se requiere para la expresión total de la hipofagia inducida por la administración intrahipotalámica de 5-HT y que la contribución de estos receptores involucra mecanismos conductuales específicos, afectando la ingesta y duración de carbohidratos.

Palabras clave: 5-HT, ketanserina, ingesta de alimento, estructura de la conducta alimentaria.

\begin{abstract}
There is evidence that the serotonergic control of feeding behavior is mediated mainly by specific $5-\mathrm{HT}_{1 / 2}$ receptors. However, there are not enough data about the specific behavioral mechanisms involved. The aim of present study was to evaluate the effects of 5-HT injection in ketanserin-pretreated rats on feeding behavior structure, in order to reveal the contribution of the $5-\mathrm{HT}_{2 \mathrm{~A} / 2 \mathrm{C}}$ receptors of paraventricular nucleus (PVN) of hypothalamus.and the specific behavioral mechanisms related. Forty rats were maintained on a self-selection feeding paradigm and provided with freely available and separate sources of protein, carbohydrate and fat. The structure of feeding behaviour was videorecorded during a period of $20 \mathrm{~min}$. The experimental room where the rats were observed was kept at $21 \pm 1^{\circ} \mathrm{C}$ on a reversed 12-h light/dark cycle. Our results show that ketanserin-pretreatment attenuated the suppressive effect of 5-HT on carbohydrate intake, even at the onset of the natural (dark) feeding period and it was observed an increase in the duration of carbohydrate meals. The ketanserin-pretreatment was unable to prevent the decrease on local eating rate induced by $5-\mathrm{HT}$. Our results strongly suggested that stimulation of $5-\mathrm{HT}_{2 \mathrm{~A} / 2 \mathrm{C}}$ receptor is required to the full expression of 5-HT-induced hypophagia, and the contribution of these receptors involves specific behavioral mechanisms, affecting carbohydrates intake and duration.
\end{abstract}

Keywords: 5-HT, ketanserin, food intake, structure of feeding behavior.

Ya ha sido ampliamente demostrado que la serotonina (5-HT) disminuye la ingestión de alimento. La 5-HT es sintetizada en el núcleo de rafé y liberada en varias estructuras hipotalámicas. Los fármacos que facilitan la transmisión serotoninérgica disminuyen la ingestión de alimentos (Leibowitz, Weiss \& Suh, 1990; Klodzinska \& Chojnacja-Wojcik, 1990; Kitchener \& Dourish, 1994; Leibowitz \& Alexander, 1998), afectando principalmente el consumo 
de carbohidratos (Wurtman \& Wurtman, 1977; Wurtman \& Wurtman, 1979a; Wurtman \& Wurtman, 1979b). También se ha reportado que tanto antagonistas como depletores de la 5-HT estimulan la ingestión de alimento (Breisch, Zemian \& Hoebel, 1976; Clineschmidt, McGuffin \& Werner, 1974; Saller \& Stricker, 1976). Sin embargo, los subtipos de receptores a serotonina responsables del control serotoninérgico de la conducta alimentaria, continúan siendo controvertidos.

Distintos subtipos de receptores serotoninérgicos han sido caracterizados y clasificados dentro de siete familias (Conn \& Sanders-Bush, 1987; Peroutka, 1988; Hoyer, et al., 1994), pero la evidencia específica señala que la conducta alimentaria es principalmente afectada por los receptores $5-\mathrm{HT}_{1 \mathrm{~A}}$ (Pedigo, Yamamura \& Nelson, 1981; Dourish, Clark, Fletcher \& Iversen, 1989), 5- $\mathrm{HT}_{1 \mathrm{~B}}$ (Peroutka, 1986); 5- $\mathrm{HT}_{2 \mathrm{C}}$ (Hoyer, Ángel \& Kalkman, 1985); 5$\mathrm{HT}_{1 \mathrm{D}}$ (Heuring \& Peroutka, 1987); 5- $\mathrm{HT}_{2}$ (formalmente 5- $\mathrm{HT}_{2 \mathrm{~A}}$; Peroutka \& Snyder, 1979) y $5-\mathrm{HT}_{3}$ (Peroutka, 1988).

El rol que juegan estos subtipos de receptores en relación a la conducta alimentaria ha sido evaluado. El agonista de los receptores $5-\mathrm{HT}_{1 \mathrm{~A}}(8-\mathrm{OH}-$ DPAT) estimula la ingestión de alimento (Dourish, Hutson \& Curzon, 1985a; 1985b; 1986; Gilbert \& Dourish, 1987; Hutson, Dourish \& Curzon, 1986; 1988), inhibiendo el disparo de las células 5-HT, vía la activación de autoreceptores somatodendríticos. Por otro lado, Scheter \& Simansky (1988) sugieren que la acción anoréxica de la administración periférica de la 5-HT es mediada por receptores $5-\mathrm{HT}_{2}$. También, Hewitt Lee, Dourish \& Clifton (2002) han mostrado que la activación selectiva de los subtipos de receptores $5-\mathrm{HT}_{2 \mathrm{C}}$ suprime la ingestión de alimento; ya que el Ro 60-0175 disminuye la ingesta de alimento y acelera el avance de la secuencia de saciedad conductual, efecto que es bloqueado por el SB 242048, antagonista de los receptores $5-\mathrm{HT}_{2 \mathrm{C}}$.

La relación de los receptores $5-\mathrm{HT}_{2 \mathrm{~A}}$ en la regulación de la conducta alimentaria ha sido reportada en varios estudios. Mancilla-Diaz, Escartin-Perez, Lopez-Alonso \& Cruz-Morales (2002), mostraron que el efecto supresor de la 5-HT sobre la ingestión de alimento fue prevenido por la administración de mianserina, antagonista de los receptores $5-\mathrm{HT}_{2 \mathrm{~A} / 2 \mathrm{~B}}$. También se ha reportado que el efecto hipofágico de la $d$-fenfluramina es prevenido en ratas pretratadas con ketanserina (Hewson, Leighton, Hill \& Hughes, 1988). De acuerdo con Currie Coiro, Niyomchai, Lira \& Farahmand (2002), la hipofagia inducida por la administración de 5-HT puede relacionarse con el bloqueo de la acción del NPY vía la activación selectiva del receptor $5-\mathrm{HT}_{2 \mathrm{~A}}$; ya que han mostrado que el efecto de la administración de NPY sobre la ingestión de alimento es prevenido por el DOI, agonista de los receptores $5-\mathrm{HT}_{2 \mathrm{~A} / 2 \mathrm{C}}$ y dicha inhibición de los efectos del NPY fue revertido por la spiperona y la ketanserina.

Por otro lado, las evidencias documentales señalan la presencia de los receptores $5-\mathrm{HT}_{2 \mathrm{~A}}$ y $5-\mathrm{HT}_{2 \mathrm{C}}$ en el núcleo paraventricular hipotalámico (NPH). 
Con técnicas de autoradiografía usando $\left[{ }^{3} \mathrm{H}\right]$-spiperona, $\left[{ }^{3} \mathrm{H}\right]$-ketanserina, $\left[{ }^{3} \mathrm{H}\right]$-DOI y $\left[{ }^{3} \mathrm{H}\right]-\mathrm{MDL} 100907$, los receptores $5-\mathrm{HT}_{2 \mathrm{~A}}$ se han localizado particularmente en corteza, núcleo caudado, núcleo accumbens, tubérculo olfatorio e hipocampo (Barnes \& Sharp, 1999; Hoyer Hannon \& Martin, 2002). También ha sido confirmada su presencia en el NPH con técnicas de inmunohistoquímica en la subdivisión magnocelular del NPH (Zhang, et al., 2002). Los receptores 5- $\mathrm{HT}_{2 \mathrm{~A}}$ y su mRNA también han sido localizados en el NPH (Zhang, et al., 2004).

Los estudios autoradiográficos han usado una gran variedad de ligandos incluyendo $\left[{ }^{3} \mathrm{H}\right]-5 \mathrm{HT}$, $\left[{ }^{3} \mathrm{H}\right]$-mesulergina y $\left[{ }^{3} \mathrm{H}\right]-\mathrm{LSD}$ con los cuales se ha realizado un mapeo detallado de la distribución de los sitios de unión (binding) para receptores $5-\mathrm{HT}_{2 \mathrm{C}}$ en ratas y otras especies. Se ha detectado alta densidad de receptores en plexo coroideo, en corteza, núcleo accumbens, hipocampo, amigdala, núcleo caudado y substancia nigra (Barnes \& Sharp, 1999; Hoyer et al., 2002). Park Harrold, Widdowson \& Williams (1999), reportaron una alta densidad de receptores $5-\mathrm{HT}_{2 \mathrm{C}}$ en el NPH de ratas $(34.0 \pm 2.3$ $\mathrm{fmol} / \mathrm{mg}$ ), con técnicas de "binding" usando $\left[{ }^{3} \mathrm{H}\right]$-mesulergina.

Basados en lo anterior, es clara la asociación de los receptores $5-\mathrm{HT}_{2 \mathrm{~A} 2 \mathrm{C}}$ en la acción hipofágica de la 5-HT hipotalámica. Sin embargo, no se tienen datos acerca de la función de estos subtipos de receptores en los parámetros específicos de la estructura de la conducta alimentaria. Con el propósito de conocer los mecanismos neuroquímicos y conductuales involucrados, en el presente estudio se evaluaron los efectos de la administración intra-paraventricular de la 5-HT en ratas pretratadas con ketanserina sobre la estructura de la conducta alimentaria, así como determinar la contribución de los subtipos de receptores $5-\mathrm{HT}_{2 \mathrm{~A} / 2 \mathrm{C}}$ en la regulación serotoninérgica de la conducta alimentaria. . La ketaserina es un antagonista de los receptores $5-\mathrm{HT}_{2}$ con alta afinidad por los receptores $5-\mathrm{HT}_{2 \mathrm{~A}}$ (pKi 8.9), y por los $5-\mathrm{HT}_{2 \mathrm{C}}$ (pKi 7.0), su afinidad por los receptores $5-\mathrm{HT}_{2 \mathrm{~B}}$ es baja (pKi 5.4) (Barnes \& Sharp, 1999)

\section{MATERIALES Y MÉTODOS}

\section{Sujetos}

Se utilizaron 40 ratas macho de la cepa Wistar de 200-230 g al inicio del experimento. Los animales fueron provistos por el Bioterio de la FES-Iztacala, UNAM. Todos los procedimientos del presente estudio fueron realizados de acuerdo a la Norma Oficial Mexicana (NOM-062-ZOO-1999), Especificaciones Técnicas para la Producción, Cuidado y Uso de Animales de Laboratorio. 


\section{Dietas}

Hidratos de carbono (harina de maíz Maseca, maíz nixtamalizado, Molinos Azteca de Chalco S.A. de C.V., planta Teotihuacan), proteínas (proteína aislada de soya $91.5 \%$ marca Supro 500 E, distribuido por Protein Technologies International, S.A. of C.V. Checkerboard Square, St. Louis, MO), grasas (manteca vegetal Inca. Elaborado por Anderson Clayton \& Co. S.A. de C.V., Tultitlán, Estado de México). El agua fue enriquecida con Vitater, un suplemento vitamínico (hecho en México por Laboratorio Maver).

\section{Fármacos}

5-Hidroxitriptamina y Ketanserina (elaborados por Sigma Chemical Co., St. Louis, $\mathrm{MO}$ ) fueron disueltos en una solución de $\mathrm{NaCl}$ al $0.9 \%$ y administrados en dosis de $2 \mu \mathrm{mg} / 0.5 \mu \mathrm{ml}$. Ambos fármacos fueron gradualmente inyectados dentro del NPH a una velocidad de $0.3 \mu \mathrm{ml} \times 1 \mathrm{~min}$, usando una jeringa Hamilton de $5 \mu \mathrm{ml}$ (Hamilton Co., Reno, NV) y un catéter de polietileno. Para asegurar una difusión completa de las sustancias el microinyector permaneció un minuto adicional dentro de la cánula guía, luego fue retirada. Los fármacos se prepararon inmediatamente antes de su administración.

\section{Cirugía estereotáxica}

Los animales fueron anestesiados con hidrato de cloral (450mg/kg/ip). Una vez anestesiados, se fijaron a un estereotáxico y se les implantó una cánula (1.5 cm de longitud), $2 \mathrm{~mm}$ por arriba del NPH del lado derecho. Las coordenadas sugeridas se tomaron del Atlas estereotáxico de Paxinos \& Watson (1986), posterior a bregma $-1.40 \mathrm{~mm}$; lateral a la línea media $0.3 \mathrm{~mm}$ y de profundidad a partir de dura madre $7.7 \mathrm{~mm}$. Las coordenadas fueron corregidas previamente por ensayo y error en un grupo piloto, inyectando azul de metileno a través de la cánula guía hasta teñir el NPH. Posterior a la corrección, las coordenadas fueron las siguientes: posterior a bregma $-1.30 \mathrm{~mm}$; lateral a la línea media $0.4 \mathrm{~mm}$ y de profundidad a partir de dura madre -6.4 $\mathrm{mm}$. Finalmente se aplicaron $50.000 \mathrm{u} / \mathrm{kg}$, im de penicilina benzatínica para prevenir infecciones.

\section{Procedimiento}

Se colocaron a las ratas de manera aleatoria en cajas habitación, individuales, cada una de ellas con tres comederos y mantenidas en un ciclo invertido de luz-oscuridad de $12 \times 12 \mathrm{hr}$ con libre acceso a agua y comida. Los animales se pesaron a las 8:00 hr, una hora antes de iniciar el ciclo de oscuridad (9:00 
hr). Las ratas tuvieron acceso a una dieta de fuentes separadas para carbohidratos, proteínas y grasas. Cada nutrimento se cambió de lugar de acuerdo a un orden preestablecido, para evitar "preferencia de lugar". El tiempo bajo estas condiciones fue de una semana.

Después de lo anterior, los animales fueron sometidos a cirugía estereotáxica. Después de cuatro días, posterior a la cirugía (período de recuperación), los animales fueron asignaron aleatoriamente a cuatro grupos de diez animales cada uno (control, ketanserina, 5-HT y Ket+5-HT). Las ratas fueron inyectadas dentro de NPH con salina seguida por salina (control), salina seguida por Ketanserina (ketanserina), salina seguida por 5-HT (5$\mathrm{HT}$ ) y ketanserina seguida por 5-HT (Ket+5-HT). A todos los animales se les administraron dos inyecciones dentro del $\mathrm{NPH}$, el tiempo entre una y otra administración fue de diez minutos y eran regresados a sus cajas habitación. Una vez iniciado el periodo de oscuridad se realizó un registro de duración continua de $20 \mathrm{~min}$, al finalizar el registro se pesaron y rellenaron los comederos (cuidando de recolectar lo que cayera del comedero) para determinar el consumo de alimento

Las cajas habitación de los sujetos experimentales estaban conectadas a una computadora para recolectar los datos del registro continuo, auxiliados por un programa que permite cronometrar el momento de acceso a los comederos, al bebedero y el tiempo que permanece el sujeto en cada uno de ellos, consecuentemente también se conoce el alimento seleccionado por la rata. Al mismo tiempo se filmaron todos los sujetos a través de una cámara de circuito cerrado para bajas intensidades de luz, para cuantificar el tiempo que pasaban inactivos (descansando). Todo esto se realizó desde un cuarto contiguo para no interferir la conducta de los sujetos experimentales.

\section{Medidas conductuales}

Se consideró como un episodio alimenticio, a un período de alimentación no interrumpido por otra conducta. Los parámetros considerados a partir de esta primera definición fueron frecuencia de los episodios alimentarios y duración de estos en segundos, tiempo entre episodios alimentarios, tasa local de la alimentación, beber y dormir. La frecuencia se definió como el número de episodios alimentarios presentes en un período de registro; la duración del episodio alimentario (s) quedó definida como el tiempo total de ingestión (de cada uno de los alimentos); el tiempo entre episodios alimentarios, se definió como el tiempo que transcurre entre un episodio alimentario y otro; la tasa local de alimentación ( $\mathrm{g} / \mathrm{s}$ ) se definió como la cantidad de alimento consumido (g) entre la duración de los episodios alimentarios (s); beber fue definido como el tiempo que permaneció el sujeto en contacto con el bebedero; y descansar como el tiempo en el que el sujeto se echaba en el extremo contrario 
a los comederos y se mantenía inmóvil. Además de los parámetros microestructurales también se calculó la ingestión de proteínas, de carbohidratos, de grasas y el total ingerido en gramos. El análisis detallado de los parámetros alimentarios permite realizar registros de menor duración (20 min) en comparación con los registros de mayor duración (4 horas o monitoreo continuo).

\section{Histología}

Al finalizar las observaciones, los animales fueron perfundidos intracardialmente, primero con solución isotónica de $\mathrm{NaCl}$ al $0.9 \%$ y luego con formalina al $10.0 \%$, para la remoción del cerebro, el cual se mantuvo 7 días en formol al $10.0 \%$. Posteriormente se realizaron cortes histológicos coronales de 70 $\mu \mathrm{m}$ de espesor con un vibratomo, para luego teñirlos con la técnica de Nissl y poder así verificar el sitio de implantación de la cánula.

\section{Análisis de resultados}

Posterior a la verificación del sitio de implantación (Figura 1), los grupos quedaron conformados de la manera siguiente: grupo Control $(n=10)$, grupo 5-HT $(n=10)$, grupo ketanserina $(n=9)$ y grupo Ket+5-HT $(n=8)$.

Cada unidad de análisis (ingestión de proteínas, carbohidratos y grasas), así como cada una de los parámetros de la estructura alimentaria (tiempo entre episodios alimenticios; duración de los episodios alimenticios; frecuencia de los episodios alimenticios; tasa local de alimentación; tiempo que dedican a beber agua y tiempo que dedican a descansar) se analizaron empleando un análisis de varianza de una entrada (ANOVA) y la comparación por pares se hizo con la prueba de Tukey. El procesamiento estadístico se llevó a cabo con el paquete denominado SPSS (versión 12.0 para Windows).

\section{RESULTADOS}

Sólo se presentan los datos donde se encontraron diferencias estadísticamente significativas $(p<0.05)$.

\section{Ingestión}

El análisis reveló diferencias significativas $[F(3,33)=4.487 ; p<0.05]$ en la ingestión carbohidratos. La comparación por pares (Tukey) indicó que la administración de 5-HT disminuyó significativamente la ingestión de carbohidratos, en comparación con el grupo control y el grupo Ketanserina. En el grupo pretratado con ketanserina (Ket+5-HT), la administración de 5-HT no 


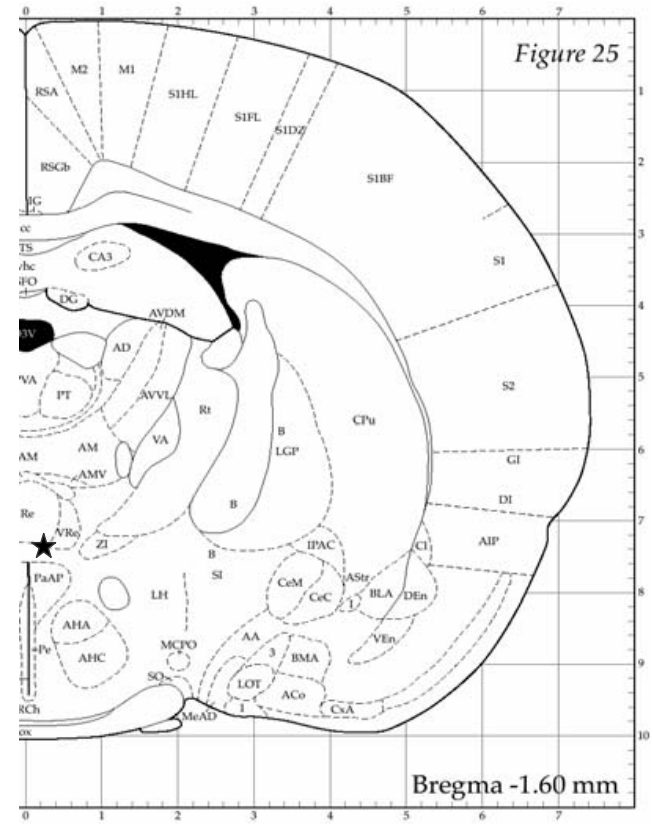

Figura 1. Posterior a la histología, las cánulas implantadas en los sujetos que se situaron en el sitio que se indica en el esquema con una estrella (tomado del atlas de Paxinos \& Watson, 1998) fueron considerados para el análisis de los datos.

mostró efectos significativos. Sin embargo, se observó que el efecto supresor de la 5-HT sobre la ingesta de carbohidratos fue atenuado por la ketanserina (Figura 2). En la ingestión de proteínas y grasas no se encontraron efectos significativos por efecto de las inyecciones intrahipotalámicas.

\section{Frecuencia}

Se encontraron diferencias en la frecuencia de los episodios alimentarios de carbohidratos $[F(3,33)=5.959 ; p<0.01]$. La comparación Post hoc mostró que la administración de 5-HT produce una disminución significativa de los episodios alimentarios de carbohidratos, en comparación con los grupos control y ketanserina. La reducción de la frecuencia de los episodios alimentarios de carbohidratos, inducida por la administración de 5-HT, no fue bloqueada por el pretratamiento de ketanserina (Ket+5-HT), ver Figura 3. La frecuencia para proteínas y grasas no fue afectada. 


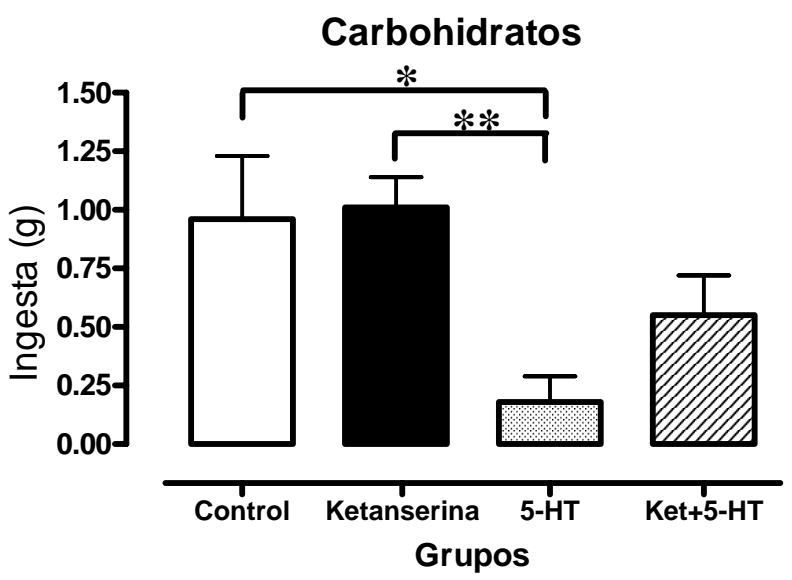

Figura 2. Los valores representan las medias \pm S.E.M. de la ingestión en gramos de carbohidratos en los cuatro grupos. ${ }^{*} p<0.05$, ${ }^{* *} p<0.01$ en la comparación por pares (Tukey).

\section{Duración}

Cuando la duración de la ingestión de carbohidratos fue analizada (Fig. 4), se encontraron diferencias entre los grupos $[F(3,33)=6.738 ; p<0.01]$. La comparación por pares mostró un aumento significativo en la duración de la ingestión de carbohidratos en el grupo Ket+5-HT, en comparación con los grupos control y 5-HT. No se observaron efectos sobre la duración en la ingestión de proteínas y grasas.

\section{Tiempo entre episodios alimentarios}

El análisis del tiempo entre episodios alimentarios mostró diferencias significativas en la comparación entre los grupos $[F(3,33)=4.074 ; p<0.05]$. En la comparación por pares se observó un aumento en el tiempo entre episodios alimentarios de la ingesta de carbohidratos del grupo 5-HT en comparación con el resto de los grupos. Sin embargo, sólo fue estadísticamente significativa la comparación grupo 5-HT vs grupo ketanserina (Fig. 5). No se observaron efectos sobre proteínas y grasas.

\section{Tasa local de alimentación}

El efecto de los tratamientos sobre este parámetro mostró diferencias significativas entre los grupos, específicamente para el consumo de carbohidratos 


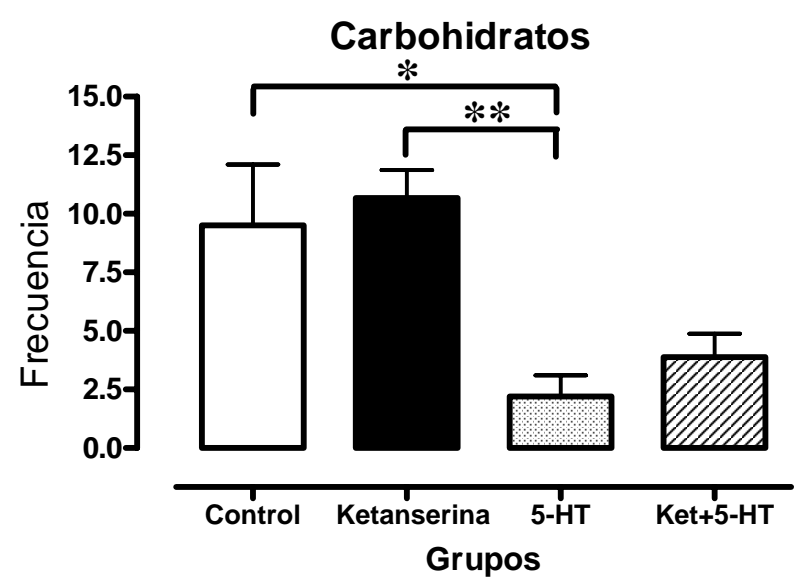

Figura 3. Los valores representan las medias \pm S.E.M. de la frecuencia en carbohidratos en los cuatro grupos. ${ }^{*} p<0.05,{ }^{* *} p<0.01$ en la comparación por pares (Tukey).

$[F(3,33)=7.916 ; p<0.001]$. En la comparación por pares se observó una disminución significativa en la tasa local de alimentación en los grupos 5-HT y ket+5-HT en comparación con el grupo control (Figura 6). No se encontraron efectos de los tratamientos sobre proteínas y grasas.

\section{Tiempo dedicado a Beber y Descansar}

El análisis estadístico (ANOVA) reveló diferencias significativas entre los cuatro grupos sobre la conducta de beber $([F(3,33)=5.219 ; p<0.01]$. En la Tabla 1 se observa una disminución significativa en el tiempo de consumo de agua en el grupo ket+5-HT, en comparación con los grupos ketanserina y 5-HT.

En el tiempo dedicado a descansar no se encontraron efectos estadísticamente significativos. Sin embargo, se observa un aumento en la conducta de descansar con la administración de la 5-HT y una tendencia a disminuir el tiempo de descansar con la administración de ketanserina (grupos ket+5$\mathrm{HT}$ ). Cabe señalar, que en estos datos se observa una alta variabilidad.

\section{DISCUSIÓN Y CONCLUSIONES}

La distribución de los receptores $5-\mathrm{HT}_{2 \mathrm{~A}}$ en el sistema nervioso central ha sido ampliamente mapeada por medio de estudios de autoradiografía e inmunocitoquímica. Usando la $\left[{ }^{3} \mathrm{H}\right]$-spiperona, $\left[{ }^{3} \mathrm{H}\right]$-ketanserina, $\left[{ }^{3} \mathrm{H}\right]-\mathrm{DOI}$ y más 


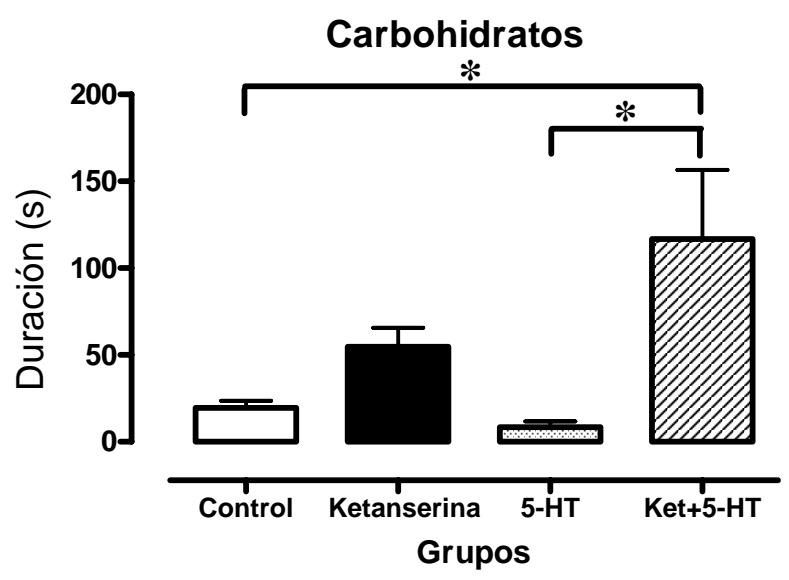

Figura 4. Los valores representan las medias \pm S.E.M. de la duración de los episodios alimentarios de carbohidratos en los cuatro grupos. ${ }^{*} p<0.01$ en la comparación por pares (Tukey).

recientemente la [ $\left.{ }^{3} \mathrm{H}\right]-\mathrm{MDL} 100907$ como radioligandos; con estudios de autoradiografía se ha reportado alta densidad de sitios de unión (binding) para los receptores $\left(5-\mathrm{HT}_{2 \mathrm{~A}}\right.$ ) en algunas regiones cerebrales (Barnes \& Sharp, 1999); particularmente en áreas corticales, núcleo caudado e hipocampo de todas las especies estudiadas (Pazos \& Palacios, 1985; Pazos, Probst \& Palacios, 1987). También se ha reportado que los sitios de unión (binding) para receptores $5-\mathrm{HT}_{2 \mathrm{~A}}$ aumentaron significativamente en el núcleo arqueado y el área hipotalámica lateral de ratas con obesidad inducida por dieta (Park et al., 1999).

Los fármacos que liberan o inhiben el metabolismo de la 5-HT, inhiben la ingestión de alimento (Blundell \& Latham, 1979; Leibowitz \& Alexander, 1998). Estos datos sugieren que los antagonistas de la 5-HT estimulan, por sí mismos, la ingestión de alimento y podrían prevenir la acción anoréxica de agentes farmacológicos que afectan a otros receptores serotoninérgicos u otros sistemas de neurotransmisión. Sin embargo, estudios previos han mostrado que la ketanserina no es capaz de bloquear totalmente la supresión de la ingestión de alimento inducida por d-fenfluramina (Grignashi, Sironi \& Samanin, 1995; Lawton, \& Blundell, 1993); la CCK-8 (Poeschla, Gibas, Simansky, Geenberg \& Smith, 1993); la fluoxetina (Lee \& Clifton, 1992) o por la leptina (Yamada, Sugimoto, Hirose \& Kajiwara, 2003). También se ha reportado que el efecto anoréxico del agonista RU 24969, agonista de los receptores $5-\mathrm{HT}_{1 \mathrm{~B}}$, no fue antagonizado por la ketanserina (Kennett, Dourish \& Curzon, 1987), aunque la hipofagia inducida por la $\alpha-m e t h y l-5-H T$ fue totalmente blo- 


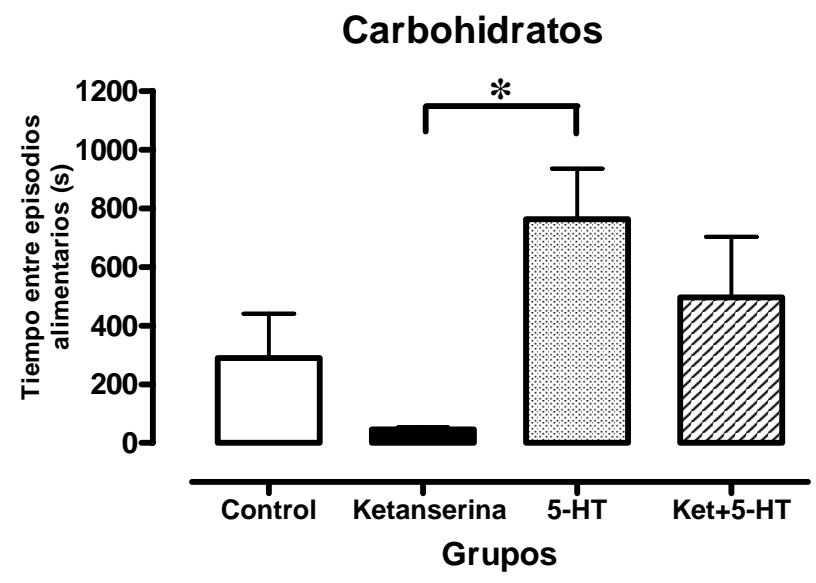

Figura 5. Los valores representan las medias \pm S.E.M. del tiempo entre episodios alimentarios de carbohidratos en los cuatro grupos. ${ }^{*} p<0.01$ en la comparación por pares (Tukey).

queada por la administración periférica de ketanserina (Sugimoto, Yamada, Yoshikawa, Noma \& Horisaka, 1996).

Adicionalmente se ha mostrado que la ketanserina atenúa la reducción en la ingestión de alimentos inducida por el TFMPP, agonista de los receptores $5-\mathrm{HT}_{1 \mathrm{~B} / 2 \mathrm{C} / 2 \mathrm{~A}}$ (Klodzinska \& Chojnacka-Wojcik, 1990) y revierte completamente el efecto anoréxico inducido por el DOI (Shechter \& Simansky, 1988).

Recientemente Hsiao, Cheng, Inui, Tong y Cheng (2006), reportaron que la administración periférica de $5-\mathrm{HT}$ reduce la hiperfagia de ratones transgénicos con sobre expresión del NPY. La administración de ketanserina fue capaz de revertir el efecto hipofágico inducido por la 5-HT, atribuyendo estos resultados a la participación de receptores $5-\mathrm{HT}_{2 \mathrm{~A}}$. Estos datos apoyan la idea de que los receptores $5-\mathrm{HT}_{2 \mathrm{~A} / 2 \mathrm{C}}$ están involucrados en la regulación serotoninérgica de la conducta alimentaria.

En el presente estudio se encontró que la administración de 5-HT en el NPH afectó algunos de los parámetros conductuales de la alimentación. La 5-HT disminuyó la ingestión de carbohidratos durante el arranque natural del periodo de alimentación (oscuridad). Estos datos son consistentes con reportes previos en los que se ha mostrado que los fármacos que facilitan la transmisión serotoninérgica disminuyen la ingestión de alimento (Leibowitz, et al., 1990; Klodzinska \& Chojnacka-Wojcik, 1990; Kitchener \& Dourish, 1994; Leibowitz \& Alexander, 1998). En el presente estudio, el efecto de la 5-HT sobre la ingestión de carbohidratos fue atenuado por el pretratamiento con ketanserina, apoyando los resultados de experimentos donde la ketanserina ha mos- 


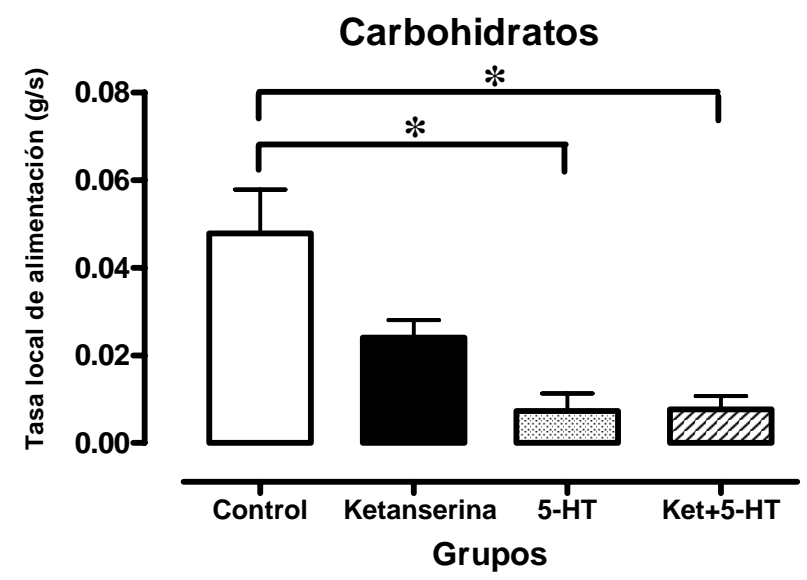

Figura 6. Los valores representan las medias \pm S.E.M. de la tasa local de alimentación de carbohidratos en los cuatro grupos. ${ }^{*} p<0.01$ en la comparación por pares (Tukey).

trado la capacidad de atenuar el efecto supresor de fármacos que facilitan la transmisión serotoninérgica, tales como la a-methyl-5-HT (Sugimoto, et al., 1996); la TFMPP (Klodzinska \& Chojnacka-Wojcik, 1990) y el DOI (Scheter \& Simansky, 1988). Sin embargo, dado que el pretratamiento con ketanserina fue incapaz de bloquear totalmente la hipofagia inducida por la 5-HT, se hace evidente la participación de diferentes subtipos de receptores a 5-HT.

Por otro lado, el pretratamiento con ketanserina no logró prevenir la disminución de la frecuencia y la tasa local de alimentación de carbohidratos, inducida por la 5-HT. A pesar de ello, para la tasa local de alimentación, la ausencia del antagonismo de la ketanserina sobre los efectos de la 5-HT indica una reducción en la cantidad de alimento consumido por unidad de tiempo; reducción que a su vez está directamente relacionada al aumento en la duración del consumo de carbohidratos producido por el pretratamiento de ketanserina; lo que podría indicar la participación indirecta de receptores $5-\mathrm{HT}_{2 \mathrm{~A} / 2 \mathrm{C}}$ en el control de la tasa local de alimentación y la duración de los episodios alimentarios para el consumo de carbohidratos.

También es posible suponer que los receptores $5-\mathrm{HT}_{2 \mathrm{C}}$ pero no los 5$\mathrm{HT}_{2 \mathrm{~A}}$ podrían ser responsables de mediar la tasa local de alimentación en el consumo de carbohidratos. De acuerdo con esto, Clifton, Lee \& Dourish (2000) reportaron que el Ro 60-0175, agonista selectivo para receptores 5$\mathrm{HT}_{2 \mathrm{C}}$ produjo la reducción de la tasa local de alimentación y en el tamaño (duración) del episodio alimentario. Estos datos son útiles para entender por qué la Ketanserina no previno el efecto de la 5-HT sobre la tasa local de ali- 
Tabla 1. Los valores representan las medias \pm S.E.M. de la conducta de beber $y$ descansar en los cuatro grupos. ${ }^{*} p<0.01$ en la comparación por pares (Tukey).

\begin{tabular}{lll}
\hline & Beber $(\mathrm{s})$ & Descansar $(\mathrm{s})$ \\
\cline { 2 - 3 } Tratamientos & & \\
\hline Control & $29.7 \pm 10$ & $25.5 \pm 25$ \\
Ketanserina & $101.11 \pm 21.3$ & $10.22 \pm 10.22$ \\
5-HT & $83.9 \pm 27$ & $310.1 \pm 152$ \\
Ket+5-HT & $6.6 \pm 3.6$ * & $54 \pm 51$ \\
\hline
\end{tabular}

mentación, ya que ketanserina ha mostrado alta selectividad para receptores 5- $\mathrm{HT}_{2 \mathrm{~A}}$ (Hoyer, et al., 1994).

Adicionalmente, el aumento sustancial en la duración del consumo de carbohidratos producido por la ketanserina y particularmente en el grupo pretratado con ketanserina (ket+5-HT), sugiere una afectación al proceso de satisfacción; ya que se observó que la cesación en el consumo de carbohidratos fue demorada. También se observó que el pretratamiento con ketanserina (grupo ket+5-HT) atenuó el aumento del tiempo entre episodios alimentarios inducido por la administración de 5-HT.

La administración de ketanserina y 5-HT no mostró efectos sobre la ingestión de grasas y proteínas ni sus parámetros alimentarios, incluyendo la conducta de descansar. Estos datos sugieren que la ingestión de grasas y parámetros alimentarios asociados, podría estar mediado por diferentes sistemas o subtipos de receptores durante el inicio del periodo natural de la alimentación (oscuridad). Para el caso de la conducta de descansar, resulta difícil la interpretación debido a la alta variabilidad de los datos.

Finalmente, los resultados de este estudio apoyan fuertemente la hipótesis de que se requiere de la estimulación de los receptores $5-\mathrm{HT}_{2 \mathrm{~A} / 2 \mathrm{C}}$ para la expresión total de la hipofagia inducida por la administración intrahipotalámica de $5-\mathrm{HT}$. Estos datos sugieren una importante participación, de los receptores $5-\mathrm{HT}_{2 \mathrm{~A} / 2 \mathrm{C}}$ en mecanismos conductuales específicos; regulando la ingestión y duración de los episodios alimentarios (proceso de satisfacción) e inhibiendo la ingestión voluntaria de carbohidratos.

\section{REFERENCIAS}

Barnes, N.M. \& Sharp, T. (1999). A review of central 5-HT receptors and their function. Neuropharmacology, 38, 1083-1152.

Blundell, J.E. \& Latham, C.J. (1979). Pharmacology of food and water intake. In S. 
Cooper \& K. Brown (Eds.) Chemical Influences on Behavior (Pp 201-254). Academic Press: London.

Breisch, S.T., Zemian, F.P. \& Hoebel, B.G (1976). Hyperphagia and obesity following serotonin depletion by intraventricular p-chlorophenyl-alanine. Science, 192, 382-385.

Clifton, P.G., Lee, M.D. \& Dourish, C.T. (2000). Similarities in the action of Ro 60-0175, a $5-\mathrm{HT}_{2 \mathrm{C}}$ receptor agonist, and $d$-fenfluramine on feeding patterns in the rat. Psychopharmacology, 152, 252-267.

Clineschmidt, B.V., McGuffin, J.C. \& Werner, A.B. (1974). Role of monoamines in the anorexigenic actions of fenfluramine, amphetamine and p-chloromethamphetamine. European Journal of Pharmacology, 27, 313-323.

Conn, P.J. \& Sanders-Bush, E. (1987). Central serotonin receptors: Effector systems, physiological roles and regulation. Psychopharmacology (Berlin), 92, 267-277

Currie, P.J., Coiro, C.D., Niyomchai, T., Lira, A. \& Farahmand, F. (2002). Hypothalamic paraventricular 5-hydroxytryptamine: receptor-specific inhibition of NPY-stimulated eating and energy metabolism. Pharmacology Biochemistry and Behavior, $71,709-716$

Dourish, C.T., Clark, M.L., Fletcher, A. \& Iversen, S.D. (1989). Evidence that blockade of $5-\mathrm{HT}_{1}$ receptors elicits feeding in satiated rats. Psychopharmacology (Berlin), $97,54-58$

Dourish, C.T., Hutson, P.H. \& Curzon, G. (1985a). Low doses of the putative serotonin agonist, 8-hydroxy-2(di-n-propylamino)tetralin (8-OH-DPAT) elicit feeding in the rat. Psychopharmacology (Berlin), 86, 197-204

Dourish, C.T., Hutson, P.H. \& Curzon, G. (1985b). Characteristics of feeding induced by serotonin agonist 8-hydroxy-2(di-n-propylamino)tetralin (8-OH-DPAT). Brain Research Bulletin, 15, 377-384.

Dourish, C.T., Hutson, P.H. \& Curzon, G. (1986). Parachlorophenylalanine prevents feeding induced by the serotonin agonist 8-hydroxy-2(di-n-propylamino)tetralin (8-OH-DPAT). Psychopharmacology (Berlin), 89, 467-471.

Gilbert, F. \& Dourish, C.T. (1987). Effects of the novel anxyolitics gepirone, buspirone and isapirone on free feeding and on feeding induced by 8-OH-DPAT. Psychopharmacology (Berlin), 93, 349-352.

Grignashi, G., Sironi, F. \& Samanin, R. (1995). The 5-HT 1 receptor mediates the of d-fenfluramine on eating caused by intra-hypothalamic injection of neuropeptide Y. European Journal of Pharmacology, 274, 221-224.

Heuring, R.E. \& Peroutka, S.J. (1987). Characterization of ${ }^{3} \mathrm{H}-5-\mathrm{HT}$ binding in bovine caudate. Journal of Neurosciences, 7, 894-903.

Hewitt, K.N., Lee, M.D., Dourish, C.T. \& Clifton, P.G. (2002). Serotonin 2C receptor agonists and the behavioural satiety sequence in mice. Pharmacology Biochemistry and Behavior, 71, 691-700.

Hewson, G., Leighton, G.E., Hill, R.G. \& Hughes, J. (1988). Ketanserin antagonizes the anorectic effect of DL-fenfluramine in the rat. European Journal of Pharmacology, 145, 227-230.

Hoyer, D., Clarke, D.E., Fozard, J.R., Hartig, P.R., Martin, G.R., Mylechrane, E.J., Saxena, P.R. \& Humprey, P.A. (1994). VII International union of pharmacology classification of receptors for 5-hydroxytryptamine (serotonin). The American Society for Pharmacology and Experimental Therapeutics, 46, 157-202. 
Hoyer, D., Engel, G. \& Kalkman, H.O. (1985). Molecular pharmacology of 5-HT, and 5$\mathrm{HT}_{2}$ recognition sites in rat and pig brain membranes: Radioligand binding studies with $\left[{ }^{3} \mathrm{H}\right] 5-\mathrm{HT},\left[{ }^{3} \mathrm{H}\right] 8-\mathrm{OH}$-DPAT, $\left.(-)\left[{ }^{125}\right]\right]$ iodocyanopindolol, $\left[{ }^{3} \mathrm{H}\right]$ mesulgerine and $\left[{ }^{3} \mathrm{H}\right]$ ketanserin. European Journal of Pharmacology, 118, 13-23.

Hoyer, D., Hannon, J. \& Martin, G. (2002). Molecular, pharmacological and functional diversity of 5-HT receptors. Pharmacology Biochemistry and Behavior, 71, 533554.

Hsiao, S-H., Cheng, H-H., Inui, A; Tong, Y-T., \& Cheng, J-T. (2006). Inhibitory effect of 5-hydroxytryptamine on hyperphagia in mice with genetic overexpression of neuropeptide Y. Neuroscience Letters, 394,256-258.

Hutson, P.H., Dourish, C.T. \& Curzon, G. (1986). Neurochemical and behavioural evidence for mediation of the hyperphagic action of 8 -OH-DPAT by $5-\mathrm{HT}$ cell body autoreceptors. European Journal of Pharmacology, 129, 347-352.

Hutson, P.H., Dourish, C.T. \& Curzon, G. (1988). Evidence that the hyperphagic response to $8-\mathrm{OH}-\mathrm{DPAT}$ is mediated by $5-\mathrm{HT}_{1}$ receptors. European Journal of Pharmacology, 150, 361-366.

Kennett, G.A., Dourish, C.T. \& Curzon, G. (1987). 5-HT ${ }_{1 \mathrm{~B}}$ agonists induce anorexia at a post-synaptic site. European Journal of Pharmacology, 141, 429-435.

Kitchener, S.J. \& Dourish, C.T. (1994). An examination of the behavioural specificity of hypophagia induced by $5-\mathrm{HT}_{1 \mathrm{~B}}, 5-\mathrm{HT}_{1 \mathrm{C}}$ and $5-\mathrm{HT}_{2}$ receptor agonist using postprandial satiety sequence in rats. Psychopharmacology, 113, 369-377.

Klodzinska, A. \& Chojnacja-Wojcik, E. (1990). Anorexia induced by M-trifluoromethylphenyl-piperazine (TFMPP) in rats. Journal of Pharmacology \& Pharmacy, 42, 17-43.

Lawton, C. L. \& Blundell, J. E. (1993). 5-HT and carbohydrate suppression: Effects of 5-HT antagonists on the action of d-fenfluramine and DOI. Pharmacology Biochemistry and Behavior, 46, 349-360.

Lee, M. D. \& Clifton, P. G. (1992). Partial reverse of fluoxetine anorexia by the 5- HT antagonist metergoline. Psychopharmacology, 107, 359-364.

Leibowitz, S.F. \& Alexander, J.T. (1998). Hypothalamic serotonin in control of eating behavior, meal size, and body weight. Biological Psychiatry, 44, 851-64.

Leibowitz, S.F., Weiss, G.F. \& Suh, J.S. (1990). Medial Hypothalamic nuclei mediate serotonins inhibitory effect on feeding behavior. Pharmacology Biochemistry and Behavior, 37, 735-742.

Mancilla-Díaz, J.M., Escartín-Pérez, R.E., López-Alonso, V.E. \& Cruz-Morales, S.E. (2002). Effect of $5-\mathrm{HT}$ in mianserin-pretreated rats on the structure of feeding behavior. European of Neuropsychopharmacology, 12, 445-451.

Park, S., Harrold, J.A., Widdowson, P.S. \& Williams, G. (1999). Increased binding at $5-\mathrm{HT}_{1 \mathrm{~A}}, 5-\mathrm{HT}_{1 \mathrm{~B}}$, and $5-\mathrm{HT}_{2 \mathrm{~A}}$ receptors and 5- $\mathrm{HT}$ transporters in diet-induced obese rats. Brain Research, 847, 90-97.

Paxinos, G. \& Watson, Ch. (1986.). The rat brain in stereotaxic coordinates. Academic Press: New York.

Pazos A, Probst A, Palacios JM (1987) Serotonin receptors in the human brain-IV. Autoradiographic mapping of serotonin-2 receptors. Neuroscience, 21, 123-139.

Pazos, A. \& Palacios, J.M. (1985). Quantitative autoradiographic mapping of serotonin receptors in the rat brain. I Serotonin-1 receptors. Brain Res 346: 205-230. 
Pedigo, N.W., Yamamura, H.I. \& Nelson, D. (1981). Discrimination of multiple $\left[{ }^{3} H\right] 5-$ hydroxytryptamine binding sites by the neuroleptic spiperone in rat brain. Journal of Neurochemistry, 36, 220-226.

Peroutka, S.J. \& Snyder, S.H. (1979). Multiple serotonin receptors: Differential binding of ${ }^{3} \mathrm{H}$-serotonin, ${ }^{3} \mathrm{H}$-lysergic acid diethylamide and ${ }^{3} \mathrm{H}$-spiroperidol. Pharmacology, 16, 687-699.

Peroutka, S.J. (1986). Pharmacological differentiation and characterization of $5-\mathrm{HT}_{1 \mathrm{~A}}$, $5-\mathrm{HT}_{1 \mathrm{~B}}$ and $5-\mathrm{HT}_{1 \mathrm{C}}$ binding sites in rat frontal cortex. Journal of Neurochemistry, 47, 529-540.

Peroutka, S.J. (1988). 5-hydroxytryptamine receptor subtypes. Annals Review of Neuroscience, 11, 45-60.

Poeschla, B., Gibbs, J., Simansky, K.J., Geenberg, D. \& Smith, G.P. (1993). Cholecystokinin-induced satiety depends on activation of $5-\mathrm{HT}_{1 \mathrm{C}}$ receptors. American Journal of Physiology, 264, 62-64.

Saller, C.F. \& Stricker, E.M. (1976). Hyperphagia and increased growth in rats after intraventricular injection of 5-7-dyxydroxytriptamine. Science, 192, 385-387.

Scheter, L.E. \& Simansky, K.J. (1988). 1-(2,5-Dimethoxy-4-iodophenyl)-2-aminopropane (DOI) exerts an anorexic action that is blocked by $5-\mathrm{HT}_{2}$ antagonists rats. Psychopharmacology, 94, 342-346.

Sugimoto, Y., Yamada, J., Yoshikawa, T., Noma, T. \& Horisaka, K. (1996). Effects of peripheral $5-\mathrm{HT}_{2}$ and $5-\mathrm{HT}_{3}$ receptor agonists on food intake in food-deprived and 2-deoxy-D-glucose-treated rats. European Journal of Pharmacology, 316, 15-21.

Wurtman, J.D. \& Wurtman, R.J. (1977). Fenfluramine and fluoxetine spare protein consumption while suppressing caloric intake by rats. Science, 198, 1178-1180.

Wurtman, J.D. \& Wurtman, R.J. (1979a). Drugs that enhance serotonergic transmission diminish elective carbohydrate consumption by rats. Life Science, 24, 895904.

Wurtman, J.D. \& Wurtman, R.J. (1979b). Fenfluramine and other serotonergic drugs depress food intake and carbohydrate consumption while sparing protein consumption. Current Medical Research Op, 6 supplement I, 28-33.

Yamada, J., Sugimoto, Y., Hirose, H. \& Kajiwara, Y. (2003) Role of serotoninergic mechanisms in leptin-induced supresión of milk intake in mice. Neuroscience Letters, 348, 195-197.

Zhang, Y., Damjanoska, K., Carrasco, G., Dudas, B., D’Souza, D., Tetzlaff, J., García, F., Sullivan, N., Scripathirathan, K., Petersen, B., Gray, T., Battaglia, G., Muma, N. \& Van de Kar, L. (2002). Evidence that 5-HT2A receptors in the hypothalamic paraventricular nucleus mediate neuroendocrine responses to (-)DOI. Journal of Neuroscience, 22(21), 9635-9642.

Zhang, Y., Gray, T., D’Souza, D., Carrasco, G., Damjanoska, K., Dudas, B., García, F., Zainelli, G., Sullivan, N., Battaglia, G., Muma, N. \& Van de Kar, G. (2004). Desensitization of 5-HT1A receptors by 5-HT2A receptors in neuroendocrine neurons in vivo. Journal Pharmacology and Experimental Therapeutics, 310, 59-66. 